\title{
Fibroadenomas and breast carcinoma: a possible answer to a frequently asked question. A pictorial essay
}

\author{
Anca Ciurea1, Hanelore Herta ${ }^{2}$, Catalin Iacoban², Bogdan Fetica ${ }^{3}$, Liliana Rogojan², \\ Cristiana Ciortea ${ }^{2}$
}

${ }^{1}$ Department of Radiology, "Iuliu Hatieganu" University of Medicine and Pharmacy, ${ }^{2}$ Emergency County Hospital, 3"Ioan Chiricuţă" Oncologic Institute, Cluj-Napoca, Romania

\section{Abstract}

Fibroadenomas (FAs) are the most frequently encountered benign breast lesions in young women and due to the age of the patients, they are usually diagnosed by ultrasound. Although they rarely undergo malignant transformation, the patients remain concerned about the possibility of malignant transformation.

The aim of this pictorial essay is to illustrate the appearance of classic and complex fibroadenomas, to discuss the risk factors and to highlight the imaging characteristics that raise the suspicion of malignant transformation. In other words, we try to answer, according to our experience and literature data, to a frequently asked question in daily practice: "Is there any risk that my fibroadenoma will become malignant?"
\end{abstract}

Keywords: ultrasound; mammography; fibroadenoma; breast cancer

\section{Introduction}

Breast cancer is the second leading cause of cancer in women worldwide. The risk factors are diverse, from positive family history to hormonal changes, number of pregnancies, diet, and even the presence of benign breast lesions.

Fibroadenomas (FAs) are the most frequently encountered benign breast lesions in young women and due to the age of the patients, they are usually diagnosed by ultrasound. Although they rarely undergo malignant transformation, the patients remain concerned about the possibility of malignant transformation.

The aim of this pictorial essay is to illustrate the appearance of classic and complex fibroadenomas, to discuss the risk factors and to highlight the imaging characteristics that raise the suspicion of malignant trans-

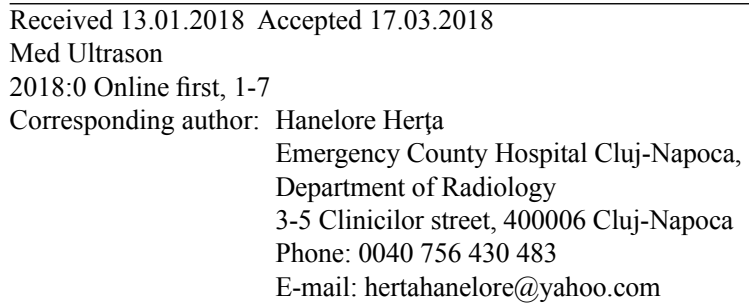

formation. In other words, we try to answer, according to our experience and literature data to a frequently asked question in daily practice: "Is there any risk that my fibroadenoma will become malignant?"

\section{Clinical examination}

FAs are the most frequent benign solid lesions of the breast, with a prevalence of $15-23 \%$ [1] and a peak incidence during the third decade and a second smaller one in the fifth decade of life [2].

Usually FAs are asymptomatic but, in up to $20 \%$ of cases [3] they can be multiple and bilateral, increasing the possibility of clinical detection at palpation. FAs are generally no larger than 2-3 cm, except the juvenile giant subtypes. Gordon et al [4] reported a safe growth rate of $20 \%$ in all three dimensions over a period of 6 months, for all age groups.

The clinical presentation may vary because, on one hand, they are hormonally responsive lesions (they grow during pregnancy/lactation and involute in menopause) and, on the other hand, during their lifetime they suffer a broad spectrum of structural changes (from highly cellular composition to hyalinization, sclerotic transformation or even calcification). 
Often at palpation, FAs are smooth, firm or rubbery, mobile and painless masses. If they undergo infarction (usually during pregnancy/lactation) they may cause pain and adherence to the surrounding tissues. If calcifications are present, they can be very firm at palpation.

\section{Pathology}

On pathology, FAs are classified as fibroepithelial tumours. On gross examination they are round-oval, well-defined, encapsulated and usually lobulated lesions, clearly delineated from the surrounding tissues [5].

On microscopic examination, FAs are an admixture of stromal and cellular elements and arise from the terminal ductolobular unit, meaning that they are composed from stromal and epithelial cells normally found in the breast. FAs that have in their structure cysts larger than $3 \mathrm{~mm}$, areas of sclerozing adenosis, epithelial calcifications or papillary changes are classified as complex FAs [6].

\section{Imaging}

FAs may exhibit a range of different imaging characteristics, from classic appearance to complex forms, representing the most common cause of biopsy in adolescents and young females.

The classical mammographic appearance of a FA is a round-oval, well defined mass, possibly lobulated, isodense to the surrounding parenchyma and sometimes hard to distinguish from the dense breast tissue (fig 1). Calcifications are often found within a degenerated FA and are subdivided into two classical benign patterns: peripheral, "rim" like calcifications, elliptical in shape and central, coarse calcifications with "popcorn" appearance [7] (fig 2). Sometimes in the early development of epithelial calcification, it can be challenging to distinguish them from the subtle, punctate pleomorphic malignant calcifications, especially when an associated mass is not visible. If the benign calcification patterns are present, no further follow-up is needed [3].

Magnetic resonance imaging (MRI) features is correlated with pathology. The high cellular FAs, also called myxoid, have higher water content showing hypointense signal on $\mathrm{T} 1$ weighted images and hyperintense signal on T2 weighted images. Sometimes the differentiation between a myxoid FA and a cystic lesion can be made only in the presence of fine internal septations within the FAs, easily depicted on T2 sequences [8]. The fibrotic FAs have poor water content, showing isointense $\mathrm{T} 1$ signal and hypointense $\mathrm{T} 2$ signal. There are also partially fibrotic FAs, with hypointense T2 signal dropping only in the sclerotic areas. The enhancement pattern also follows the

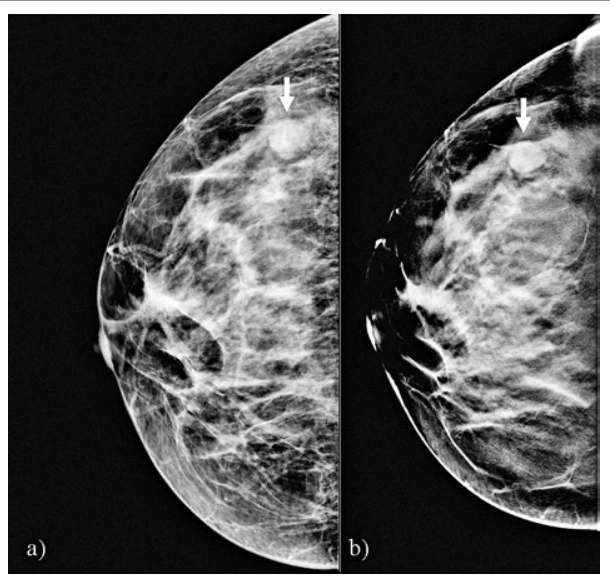

Fig 1. Mammographic appearance of a fibroadenoma (arrows) on 2D (a) and 3D (mammography): oval, well defined mass, isodense to the surrounding parenchyma.

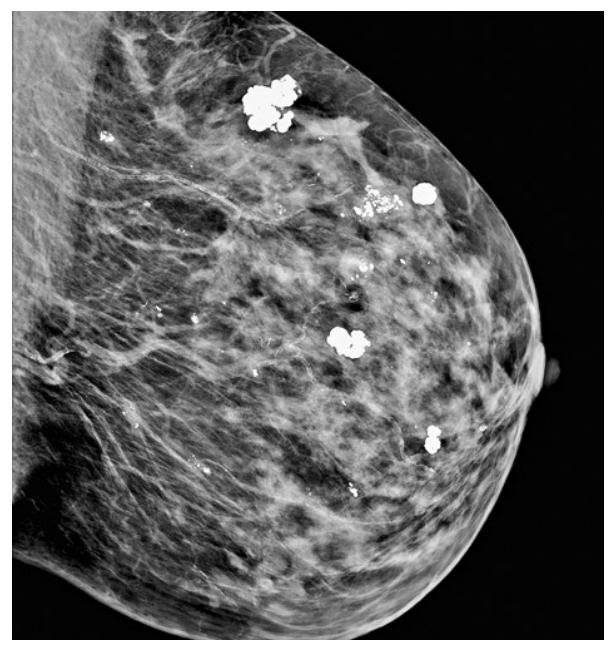

Fig 2. Multiple fibroadenomas on mammography, with typical "pop corn" calcifications.

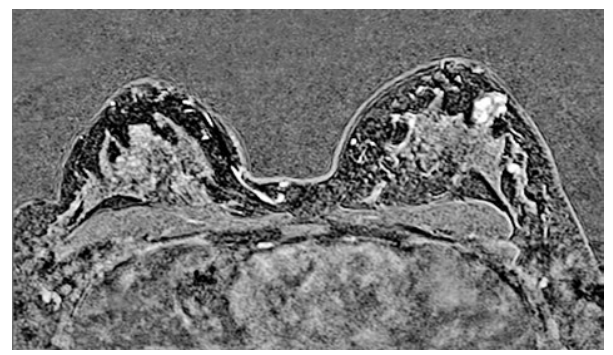

Fig 3. MRI (T1 contrast enhanced, substraction image) shows in the left breast a typical fibroadenoma: an oval enhancing mass with hypoenhancing septations.

histologic subtypes, the myxoid masses typically showing rapid and strong enhancement, due to their high cellular component, conversely to fibrotic masses that show 


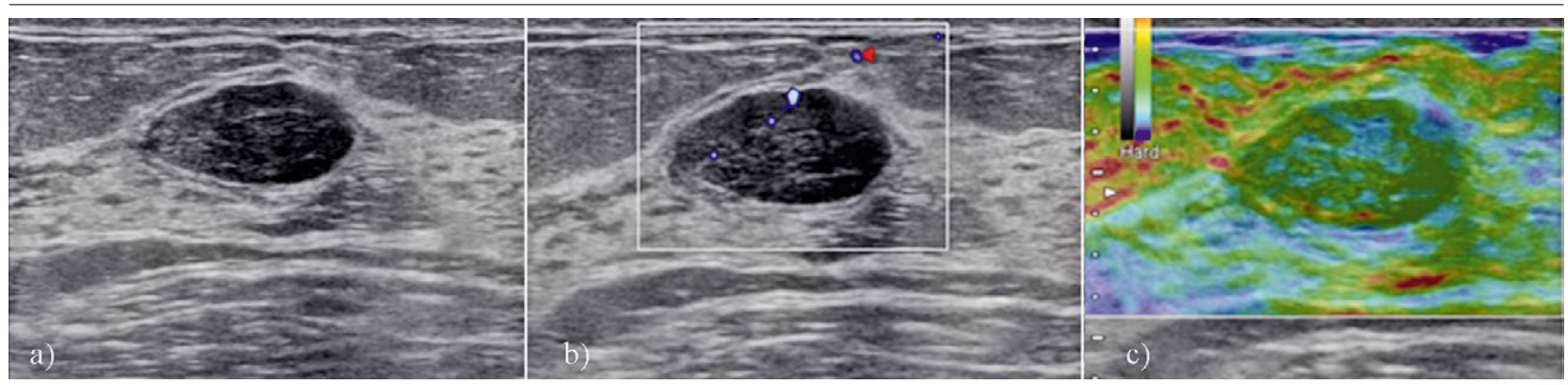

Fig 4. Typical ultrasound appearance of a fibroadenoma: oval circumscribed hypoechoic mass, with the long axis parallel to the skin, homogeneous, surrounded by a thin echogenic capsule (a). On Doppler examination segmental vessels are visible (b) and on sonoelastography the lesion has the same elasticity as the surrounding parenchyma (c).
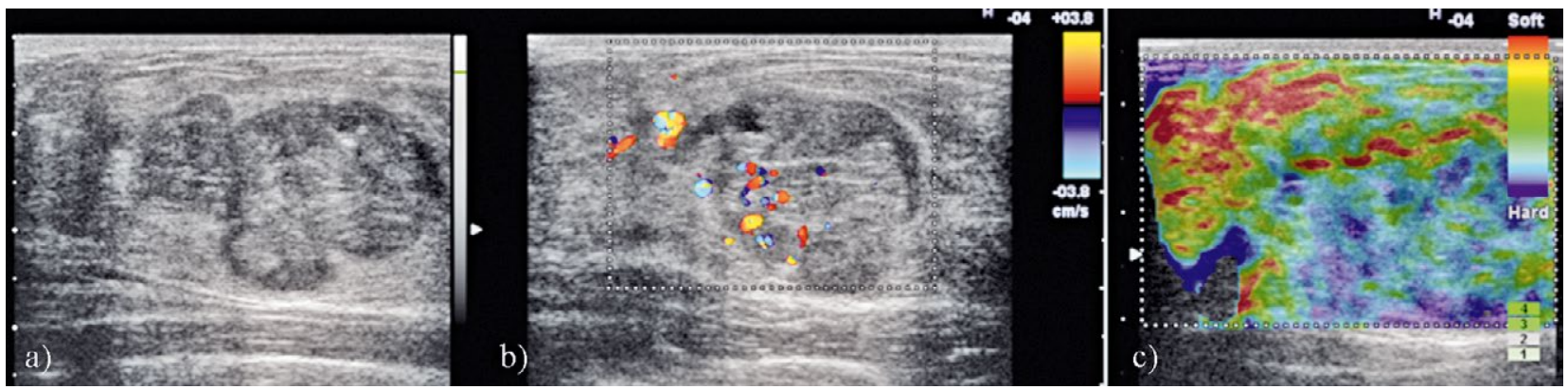

Fig 5. Complex fibroadenoma appearing on ultrasound as a lobulated mass with heterogeneous internal echotexture and small cystic inclusions (a). The lesion presents intense blood supply (b) and a mosaic pattern (score 2) on elastography (c).

little or no contrast enhancement. The internal septations are usually hypoenhancing structures [9] (fig 3).

Classical FAs appear at ultrasound (US) as parenchimal masses with benign features (fig 4). They are wellcircumscribed and oval in shape, with the long axis parallel to the skin surface, homogeneous, hypo- or isoechoic, surrounded by a thin echogenic capsule. Fine fibrous internal septations may be visible and also a few discrete lobulations. The posterior echogenicity is normal or increased, with thin edge shadows, an exception being the calcified masses that demonstrate acoustic shadowing [7]. The majority exhibit blood flow on Doppler examination, showing a feeding vessel and capsular or segmental vessels within the internal septations. Furthermore, FAs are soft, compressible masses, with uniformly low elasticity values at shear wave elastography (average $28 \mathrm{kPa}$, range 18 to $44 \mathrm{kPa}$ ) [10]. Not all FAs exhibit benign features on US, therefore further biopsy is required in order to confirm their benign nature.

Complex FAs exhibit on ultrasound less reliable characteristics (fig 5) that exclude the possibility of "probably benign"/BIRADS 3 categorization and require further biopsy. These findings include: small epithelial calcifications, often without acoustic shadowing, angular or irregular margins, heterogeneous internal echotexture and discrete cystic changes or hyperechoic foci relying on their different histologic patterns [7].

\section{FAs and breast cancer}

Being a proliferation of the normal cells of the breast, fibroadenomas may suffer the same changes such as breast parenchyma, including the development of malignancy. The literature data is limited regarding the correlation of these two entities, with a reported incidence between $0.125 \%$ and $0.02 \%$ [11].

A carcinoma within a FA is considered when the neoplastic transformations are entirely encased within the FA or when the carcinoma is focally involving the FA. There are situations when the carcinoma appears in the proximity of FAs but not within the FA, with possible consequent invasion (fig 6). Therefore, in the cases of FAs focally involved by carcinoma, the question is whether the cancer developed within or in the proximity of the FA (fig 7).

Back in 1994 DuPont et al observed that special concerns should be given to certain groups considered at risk, such as women with adjacent proliferative disease, long history of fibroadenoma (FA), breast cancer in a first-degree relative and the presence of BRCA1 gene. Also they reported an incidence of complex FAs of ap- 


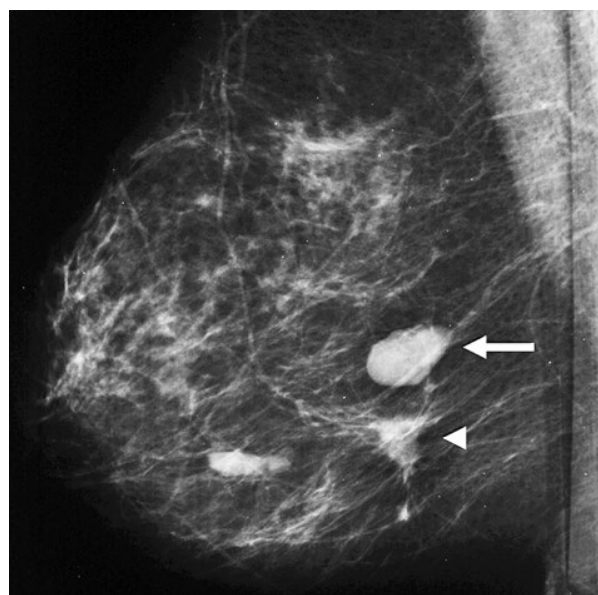

Fig 6. Mammography of the right breast. A malignant mass (short arrow) is seen in the proximity of a fibroadenoma (long arrow).

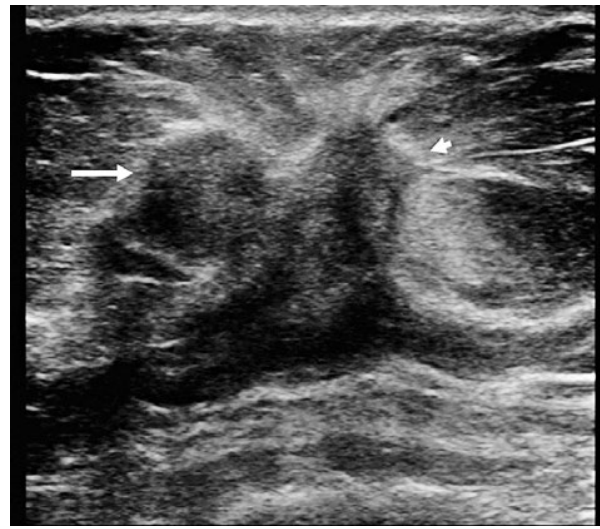

Fig 7. Ultrasound of a 57 year old patient. A lesion with benign features, histologically proved to be a fibroadenoma (long arrow) is focally involved by a pathologically proved invasive ductal carcinoma (short arrow). proximately $23 \%$ and the risk of developing bilateral carcinoma, even at distant sites, between 3.1-3.9 times higher than the general population [2].

Regarding the clinical management of these lesions there is no global consensus. In their study, Sklair-Levy et al recommended that after histological confirmation, the approach of complex FAs should be conservative as for simple FAs [12]. On the other hand, many centers are practicing lesion excision also because of the medicolegal implication of delayed breast cancer detection.

However the "in situ" forms are reported more common than invasive forms, $66.9 \%$ representing lobular carcinoma in situ (LCIS), $12.4 \%$ ductal carcinoma in situ (DCIS), $11 \%$ invasive ductal carcinoma (IDC), and 3.4\% invasive lobular carcinoma (ILC)) [13]. Malignant transformation is suggested by several altered features such as: texture, margins, shape, growth or even regression, vascularization, mobility, compressibility and surroundings.

On mammograms, the detection of new pleomorphic microcalcifications or indistinct margins within a known FA raises the suspicion of malignancy (fig 8). However, the US examination can be more helpful. It offers a more conspicuous lesion characterization such as elevated size, amorphous/irregular shape and contour, changes in echotexture, increase color-flow signals within the mass and even changes in elasticity. US can also depict new calcifications inside the FA, any new calcifications needing to be further morphologically analyzed by mammography (fig 9). Nevertheless, there are cases when a carcinoma within a FA looks indistinguishable from the complex FAs (fig 10).

In cases of malignant transformation, it is not uncommon to have two or more malignant lesions in the same or in both breasts, a fact that supports the affirmation of
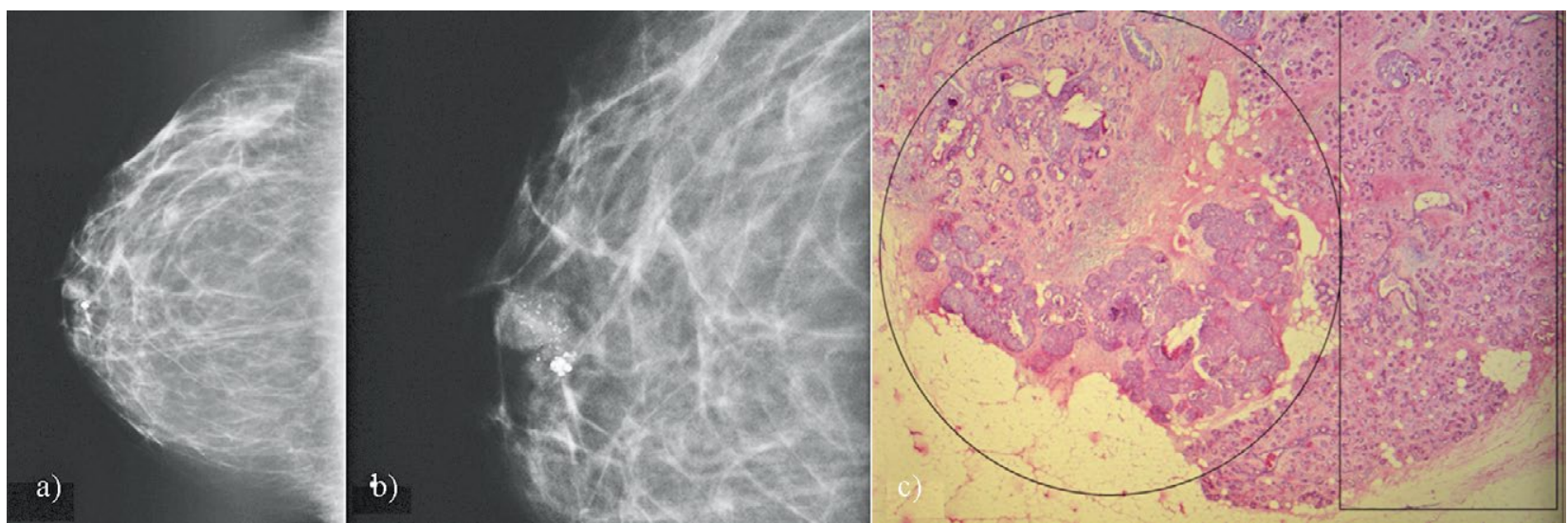

Fig 8. Mammography of the right breast in cranio-caudal projection (a) and enlarged view (b). In the retroareolar region there is a circumscribed mass with new pleomorphic associated microcalcification that raised the suspicion of associated malignancy. On the pathology specimen an IDC NST (circle) is seen inside a fibroadenoma (rectangle). 


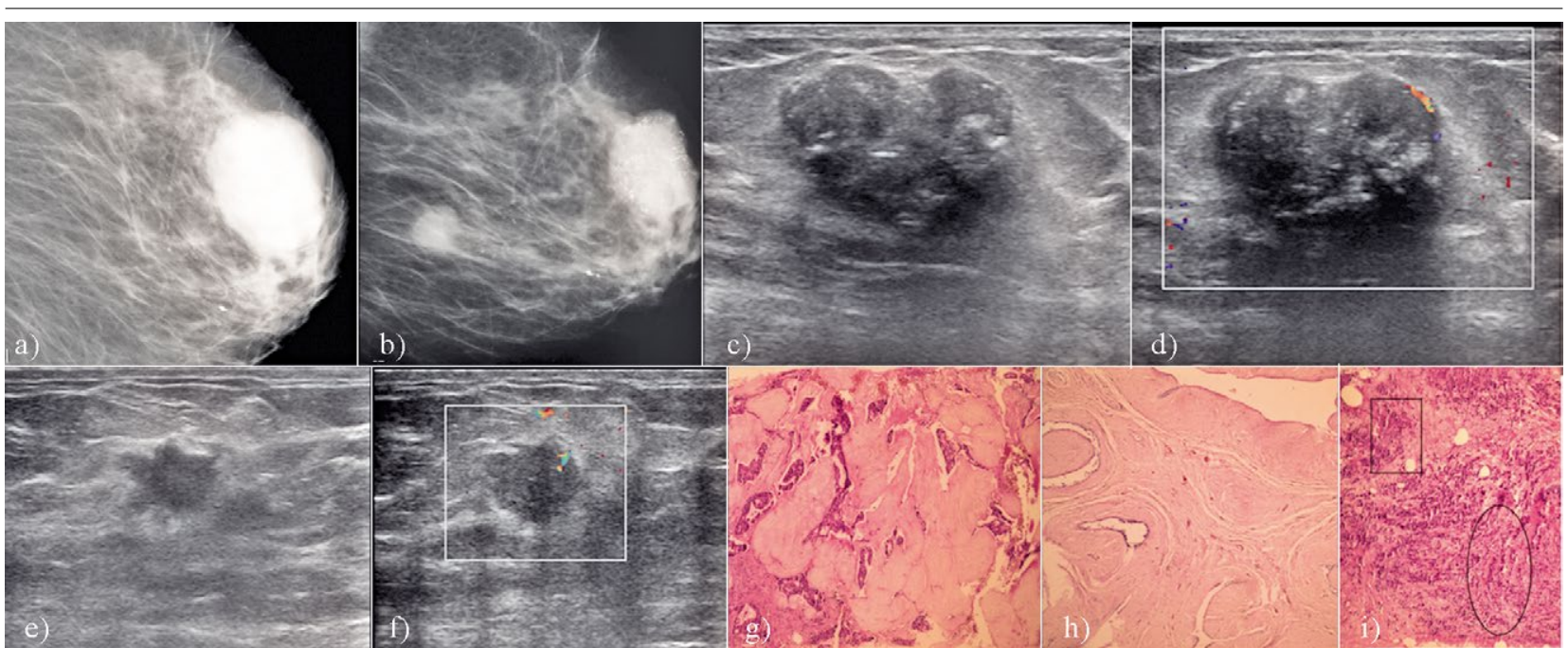

Fig 9. In a) the mammography of a 56 year old patient shows an oval, circumscribed, homogeneous mass, proved to be a fibroadenoma. Three years later, the same lesion shows dimensional regression, with newly appeared pleomorphic calcifications within the lesion (b). In the same breast, a second mass is seen, with spiculated contour, highly suggestive for malignancy. On ultrasound, the known fibroadenoma, with changed features appears as a lobulated mass, inhomogeneous due to hyperechoic spots representing the microcalcifications (c) and on Doppler ultrasound it shows circumferential vascularisation (d). The newly appeared mass is irregular and non-circumscribed, with angulated margins and hyperechoic rim (e), with plunging blood vessel (f). The pathology specimen shows an IDC with large tubular malignant structures (g) in the fibroadenoma (h) and an IDC (i) with cords (in oval) and nests (rectangle) pattern in the second, newly appeared lesion.

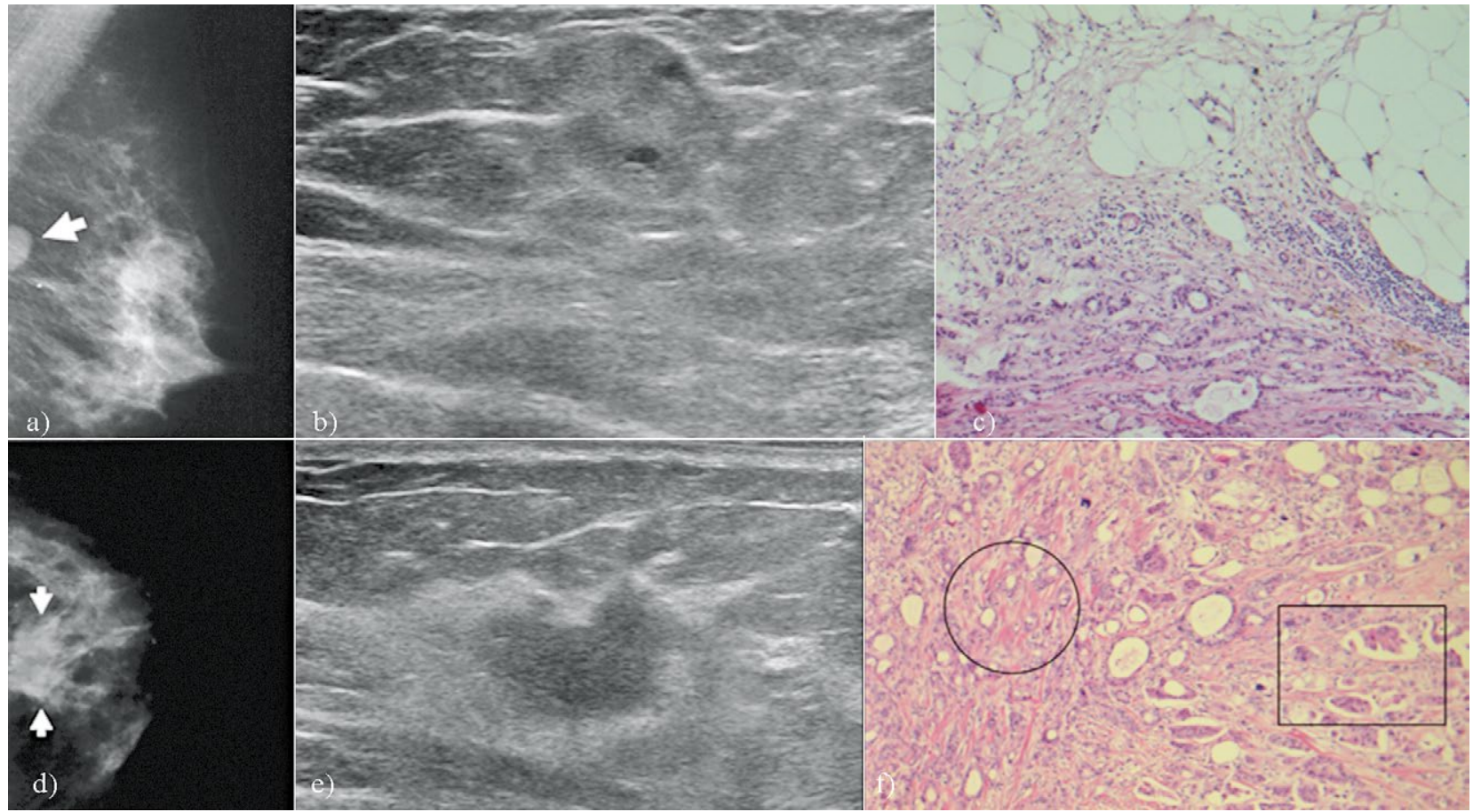

Fig 10. A 50 year old patient, with a known fibroadenoma in her left breast, presents for the screening mammography. On the mammography in medio-lateral projection (a), the fibroadenoma (arrows) presents as an oval, circumscribed mass, with benign features. On ultrasound (b), the lesion appears isoechoic, inhomogeneous due to small cystic areas inside, ill defined and oriented perpendicular to the skin, resembling a complex fibroadenoma. After the ultrasound examination the lesion was classified as suspicious and rebiopsy was indicated. At pathology, the lesion was proved to be a fibroadenoma with a IDC within (c). In the same breast, better visible on the mammography in cranio-caudal projection (d) there is a second mass, with spiculated countour. On ultrasound (e) the mass has malignant features (irregular shape, non-circumscribed contour) and after biopsy it was proved to be a NST (circle) and micropapillary (square) IDC (f). 


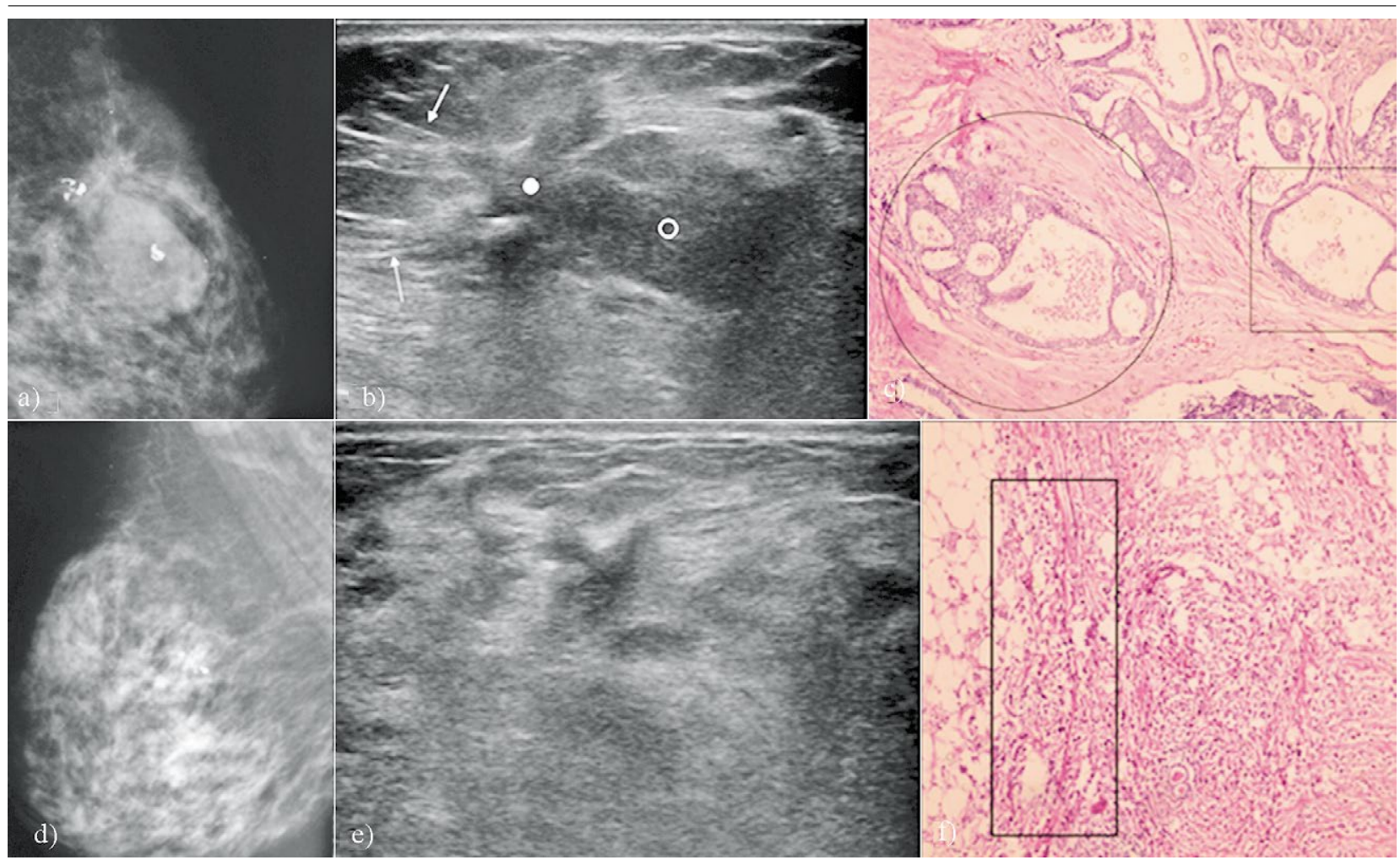

Fig 11. A 73 year old patient is referred for the diagnosis of a $10 \mathrm{~cm}$ palpable lump in her right breast. On mammography, the patient presents in her left breast (a) a circumscribed oval mass with associated benign calcifications, in the periphery with the presence of a second lesion, spiculated, with associated architectural distorsion. At ultrasound (b) a hypoechoic oval lesion with benign features in seen (circle). The lesion is in contact with and involved by a second mass (dot), with spiculated contour and architectural distortion (attraction of Cooper ligaments - arrows). The spiculated lesion in the left breast was proved to be an IDC (c) with cribriform (circle) and tubular pattern (square). In the right breast, on mammography (d), corresponding to the palpable lump, there were no significant findings but the ultrasound (e) revealed a $7 \mathrm{~mm}$ suspicious mass and a few areas of discrete shadowing. The biopsy performed from the lesion in the right breast (f) revealed an ILC with diffuse pattern (rectangle).

DuPont that women with adjacent proliferative disease are more prone to develop a breast cancer within the fibroadenoma (fig. 11) [2].

\section{Conclusion}

The management of newly detected solid lesions differs, from triple assessment to short-term follow up. These approaches allow the identification of four possible outcomes: the malignancy simulators (malignant lesions may mimic FAs), to detect rapid growth in some special histological types (juvenile FAS), to suspect the phyllodes tumors that may be identical on imaging to FAs and to diagnose the early malignant changes.

FAs, being a proliferation of normal cells of the breast, suffer the same changes as the breast itself. They can increase their size in certain physiological situations (menstruation, pregnancy, lactation), can be painful, may regress after menopause and can develop ma- lignant transformation. Even though there is a very low incidence, every radiologist should be aware of the possibility that FAs could suffer malignant alterations and give special attention to any changes that appear even in a pathologically proven FA.

\section{References}

1. Hua B, Xu JY, Jiang L, Wang Z. Fibroadenoma with an unexpected lobular carcinoma in situ: A case report and review of the literature. Oncol Lett 2015;10:1397-1401.

2. Dupont WD, Page DL, Parl FF, et al. Long-Term Risk of Breast Cancer in Women with Fibroadenoma. N Engl J Med 1994;331:10-15.

3. Chu B, Crystal P. Imaging of fibroepithelial lesions: a pictorial essay. Can Assoc Radiol J 2012;63:135-145.

4. Gordon PB, Gagnon FA, Lanzkowsky L. Solid breast masses diagnosed as fibroadenoma at fine-needle aspiration biopsy: acceptable rates of growth at long-term follow-up. Radiology 2003;229:233-238. 
5. Goel NB, Knight TE, Pandey S, Riddick-Young M, de Paredes ES, Trivedi A. Fibrous lesions of the breast: imagingpathologic correlation. Radiographics 2005;25:1547-1559.

6. Neal L, Tortorelli CL, Nassar A. Clinician's guide to imaging and pathologic findings in benign breast disease. Mayo Clin Proc 2010;85:274-279.

7. Stavros AT (ed). Breast Ultrasound. Lippincott Williams \& Wilkins, 2004:1030.

8. Fischer U (ed). Practical MR Mammography: High-Resolution MRI of the Breast. Thieme, 2012:392.

9. Park EK, Cho KR, Seo BK, et al. Radiologic Findings of Ductal Carcinoma in Situ Arising Within a Juvenile Fibroadenoma: Mammographic, Sonographic and Dynamic Contrast-Enhanced Breast MRI Features. Iran J Radiol 2015;12:e17916.
10. Wang ZL, Li JL, Li M, Huang Y, Wan WB, Tang J. Study of quantitative elastography with supersonic shear imaging in the diagnosis of breast tumours. Radiol Med 2013;118:583590.

11. Ooe A, Takahara S, Sumiyoshi K, Yamamoto H, Shiba E, Kawai J. Preoperative diagnosis of ductal carcinoma in situ arising within a mammary fibroadenoma: a case report. Jpn J Clin Oncol 2011;41:918-923.

12. Sklair-Levy M, Sella T, Alweiss T, Craciun I, Libson E, Mally B. Incidence and management of complex fibroadenomas. AJR Am J Roentgenol 2008;190:214-218.

13. Wu YT, Chen ST, Chen CJ, et al. Breast cancer arising within fibroadenoma: collective analysis of case reports in the literature and hints on treatment policy. World J Surg Oncol 2014;12:335. 